(Aus dem Pharmakologischen Institut der Universität zu Bonn.)

\title{
Der Wein als Erregungsmittel beim Menschen.
}

\author{
Von \\ Dr. J. Weissenfeld.
}

(Mit 1 Textfigur.)

Im Verfolg der Untersuchungen über den Weinge ist als Arzneimittel, die in hiesigen Pharmakologischen Institute seit 1869 unternommen wurden, handelte es sich auch um die wichtige Frage, wie sich die Athmung ihm gegenüber verhalte.

Die nachstehende Abhandlung kann als Fortsetzung und Frgänzung derjenigen gelten, die C. Wilmanns.) und G. Vogel ${ }^{2}$ ) in diesem Archiv veröffentlicht haben.

Beide Abhandlungen enthalten noch Lücken. Die Versuche sind mit Ausnahme von XXII und XXIII bei Wilmanns ${ }^{3}$ ) nur an Thieren angestellt, und es ist desshalb durchaus erforderlich, eine grössere Zahl am Menschen zu wiederholen. Ferner muss die Frage experimentell erörtert werden, ob nicht der bedeutenden St eig e rung der Athemgrösse durch den Weingeist nach einiger Zeit eine Rückwirkung in Form eines Abfalles unter die Norm folge, so dass jene Steigerung dadurch mehr als aufgewogen würde. Das war zwar nach den bisherigen B eobach tungen nicht wahrscheinlich, allein es war doch nicht ganz ausgeschlossen.

Als erste Versuchsperson bei diesen neuen Versuchen diente ich. Bin 23 Jahre alt, $83 \mathrm{~kg}$ schwer, gesund und kräftig und an den sehr mässigen Genuss von Alkoholicis gewöhnt.

Die Versuche wurden in derselben Weise angestellt, wie Wilmanns und die anderen Autoren sie schon beschrieben haben. Als Weingeistpräparat diente diesmal ein ungefähr 30 Jahre alter Xeres -

1) C. Wilmanns, Die directe Erregung der Athmungscentra durch den Weingeist Bd. $66 \mathrm{~S}$. 167. Bonn 1897.

2) G. Vogel, Untersuchungen über die Wirkung einiger Säureäther Bd. 67 S. 141. Bonn 1897.

3) Vgl. Wilmanns S. 204-206. 
w ein von sehr angenehmem Geruch und Geschmack und einem Gehalt an Weingeist von 13,7 Gewichtsprocent.

Die Versuche wurden meistens Morgens früh gegen 8 Uhr begonnen. Ich lag bequem auf einer Matratze, den Kopf erhöht, hatte das Mundstück der Gasuhr zwisehen den Lippen und Zähnen. Die Nase war durch Wattebäusche, die mit Vaseline bestrichen waren; dicht verschlossen. Das Athmen geschah durch die bekannten Ventile, Anfangs zwar mit Schwierigkeit, weil das ungewohnte Mundstück der Gasuhr Beschwerden machte. Es waren desshalb auch bei den ersten Versuchen, die angestellt wurden, meine normale Athemgrösse zu bestimmen, die Zahlen verhältnissmässig hoch; später jedoch, als ich mich an diese Art des Athmens gewöhnt hatte, ging Alles ohne jegliche Schwierigkeit, wenn nur die Ventile in bester Ordnung waren.

Im Ganzen habe ich 74 Einzelversuche in 14 Versuchsreihen mit dem Xereswein gemacht. Da alle ohne Ausnahme dasselbe Ergebniss hatten, so darf ich wohl der Kürze halber diejenigen auswählen, die dies Ergebniss am klarsten zeigen.

Zuerst wurde meine normale Athemgrösse bestimmt. Jeder Versuch dauerte 15 Minuten, alle halben Minuten wurde an der Gasuhr abgelesen und wurden die. Werthe notirt. Sobald 30 Werthe aufgeschrieben waren, wurden sie addirt und der Mittelwerth aus ihnen gezogen. Diese Mittelzahl gibt also an, wie viel Liter Luft durchschnittlich in jeder halben Minute ausgeathmet worden sind.

Der Kürze wegen gebe ich die vielen Einzelzahlen nicht, wie Wilmanns und Vogel das thaten. Ich verweise auf ilıre Darstellung, der ich mich in allem anschloss. Die arithmetischen Mittel dürften diesmal genügen. Dazu mache ich noch besonders die Angabe, dass alle Versuche in der annähernden Gleichmässigkeit verliefen wie dort.

\section{Versuch I.}

30 Aufzeichnungen gaben im Mittel eine A themgrösse von 5,92 1 in der halben Minute Athemluft. Ls war der erste Versuch. Das Mundstück war mir zwischen Lippen und Zähnen sehr unbequen.

In den folgenden Normalversuchen II, III, IV, V, VI, VII, VIII, IX verschwand diese Empfindung immer mehr und das fand seinen Ausdruck in den Mittelwerthen von

$$
\begin{array}{lllllllll}
3,24 & 2,74 & 2,96 & 2,80 & 2,96 & 2,86 & 2,96 & 2,96 & \text { I }
\end{array}
$$

Es musste ebenfalls untersucht werden, ob nicht die Aufnahme der Flüssigkeit für sich allein in den Magen die Athemgrösse vor- 
ubergehend beeinflusse. $\mathrm{Zu}$ diesem $\mathrm{Zwecke}$ machte ich eigens einen Versuch, indem ich die Athemgrösse maass und darauf $100 \mathrm{ccm}$ Wasserleitungswasser trank.

Die Zahlen waren vor der Aufnahme des Wassers im Durchschnitt 3,15 und 3,16, und nach der Aufnahme 2,65 und 2,85. Es war nicht nur nicht eine Steigerung, sondern ein Abfall erfolgt.

Auch dieser Controlversuch wurde in den nämlichen Vormittagsstunden wie die früheren gemacht.

Auf diesen Grundlagen konnte man mit der Beantwortung der Frage nach der Wirkung des Weines beginnen. Dabei verfuhr ich folgendermaassen:

Zuerst wurde die physiologische Athemgrösse aufgenommen, dann der Wein getrunken, eine Viertelstunde gewartet, um dessen Aufsaugung abzuwarten, und dann sein Einfluss auf die Athemgrösse aufgezeichnet.

Hier ein Beispiel:

\section{Versuch Ir.}

7 Uhr 40 Min. Morgens. Zimmerwärme $14^{\circ} \mathrm{C}$.

Ruhige Athmung vor der Weingeistaufnahme:

\begin{tabular}{rrrrrr}
2,7 & 3,1 & 2,3 & 2,8 & 3,4 & 2,6 \\
3,0 & 3,2 & 3,0 & 2,8 & 2,8 & 2,8 \\
3,1 & 3,1 & 3,2 & 3,4 & 2,8 & 2,8 \\
3,6 & 2,9 & 2,9 & 3,3 & 2,7 & 3,0 \\
3,1 & 2,7 & 2,6 & 2,9 & 3,3 & 3,0 \\
\hline 15,5 & 15,0 & 15,0 & 15,2 & 15,0 & 14,2
\end{tabular}

Mittlere Grösse der Athmung 2,96 I. $8 \mathrm{Uhr}$ Aufnahme von $50 \mathrm{ccm}$ Xereswein: Es wurden 10 Minuten gewartet und weitere 3 Minuten ohne Notirung vorgeathmet. Sofortiges Aufsteigen der Athemgrösse auf 3,70 $1 \mathrm{im}$ Durchschnitt. Athemgrösse um 8 Uhr 18 Min.:

\begin{tabular}{rrrrrr}
4,3 & 6,6 & 2,9 & 4,0 & 3,3 & 4,0 \\
4,0 & 3,9 & 4,1 & 3,8 & 3,1 & 3,3 \\
4,1 & 2,9 & 4,0 & 3,6 & 4,3 & 3,5 \\
3,4 & 3,3 & 3,2 & 3,9 & 3,3 & 3,9 \\
4,6 & 4,3 & 3,2 & 4,0 & 3,0 & 2,9 \\
\hline 20,4 & 21,0 & 17,4 & 19,3 & 17,0 & 17,6
\end{tabular}

Es wurde nun alle Viertelstunde abgelesen und Folgendes gewonnen:

8 Uhr 40 Min. fühlte ich mich recht schläfrig, ich war ruhig auf der Matratze liegen geblieben.

Die Athemgrösse war gestiegen auf 4,57 1 . 
9 Uhr 10 Min. war sie auf 3,801 gestiegen.

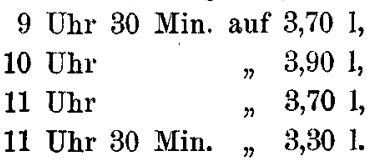

Um 10 Uhr war ich nahe am Einschlafen.

Also vor der Weingeistaufnahme wurden durchschnittlich jede halbe Minute 2,96 l Luft ausgeathmet, 4 Stunden später 3,30 1. mithin in dieser langen Zeit kein Abfall unter die Norm.

Man könnte noch an eine ähnliche Schwankung der Athemgrösse lenken, wie sie tagsüber bei der thierischen Körperwärme stattfindet und hier bis zu $0,6^{\circ}$ beträgt. Die im Versuch I angegebenen Mittelwerthe liegen jedoch sämmtlich unter 3,30 l und somit muss diese Ziffer jedenfalls angesehen werden als ni $\mathrm{cht}$ unter der Norm liegend.

\section{Versuch III.}

Dieser Versuch fand auch in derselben Weise wie die zuerst angeführten statt. Während desselben trat auch ein vollständiges Einschlafen ein. Dabei fiel die A themgrösse auf 3,50 l, also immer noch nicht a uf oder unter die Norm, von der der Versuch ausgegangen war. Wir werden auch an späteren Versuchen sehen, dass auch im schläfrigen Zustande ein Wiederansteigen der Athemgrösse vorkommt.

Der Versuch begann 8 Uhr 50 Min. Morgens.

Mittlere Grösse der Ausatmung vor Aufnahme des Weingeistes 3,10 I Luft. Nach Aufnahme des Xeresweines 9 Uhr 8 Min.; schon 9 Uhr 20 Miu. ein Aufsteigen der Athemgrösse auf 4,83 l.

9 Uhr 40 Min., während ich schlief, fiel dieselbe auf 3,50 l.

$$
\begin{array}{lllllll}
10 & \text { Uhr } & 13 & \text { Min. auf } & 3,37 & 1 \\
10 & \# & 43 & \# & \# & 3,35 & 1, \\
11 & & 15 & \# & \# & 3,45 & 1 .
\end{array}
$$

11 Uhr 42 Min. oder $2^{1 / 2}$ Stunde nach Aufnahme des Weingeistpräparates noch folgende Werthe:

\begin{tabular}{rrrrrr}
3,1 & 2,9 & 3,5 & 4,0 & 3,9 & 3,1 \\
4,0 & 2,9 & 3,3 & 3,0 & 4,3 & 3,0 \\
3,0 & 3,2 & 2,7 & 3,5 & 2,6 & 3,0 \\
4,0 & 3,8 & 3,4 & 3,4 & 2,8 & 3,0 \\
3,2 & $\mathbf{3 , 6}$ & 3,5 & 4,5 & 3,5 & 3,0 \\
\hline 17,3 & 16,4 & 16,4 & 18,4 & 17,1 & 15,1
\end{tabular}

oder im Mittel 3,30 l, ebenfalls kein Abfall unter die Norm, da ja die physiologische Athemgrösse bei Versuch III im Mittel 3,10 1 ergeben hatte.

Um eigens die Wirkung der Aussentemperatur mit in Rechnung zu ziehen, wurde diese während eines ganzen Versuches beobachtet 
und gefunden, dass sie einer Schwankung nicht unterlag, und dass der Ausschlag, den der Wein gab, sich dennoch einstellte.

Es blieb sich für das Ergebniss auch gleich, ob die Zimmerwärme die gewöhnliche $\left(17-18^{\circ} \mathrm{C}\right.$.) war, oder an Sommertagen bis zil $25^{\circ} \mathrm{C}$. betrug, oder einmal nur $14^{\circ} \mathrm{C}$.

Versuch IV.

In diesein und den folgenden Versuchen nahm ich statt $50 \mathrm{ccm}$ Wein $75 \mathrm{ccm}$ auf. Ich erhielt in der Norm, bei zwei Versuchen, 2,93 1 und 2,81 1 in der halben Minute.

9 Uhr 20 Min. wurde der Wein aufgenomuen und 9 Uhr 35 Min. die ctwaige Aenderung der Athemgrösse untersucht.

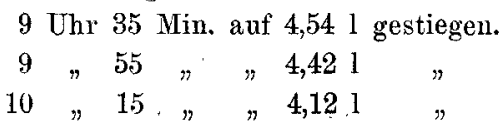

I'm 12 Uhr 15 Min. stand die Athemgrösse noch auf 3,95 l. Es erguben sich hier folgende Werthe:

\begin{tabular}{rrrrrr}
5,5 & 5,8 & 3,9 & 2,8 & 3,6 & 3,3 \\
4,7 & 3,9 & 4,0 & 5,7 & 2,5 & 3,6 \\
4,6 & 3,7 & 4,5 & 4,2 & 3,7 & 3,2 \\
5,4 & 2,8 & 4,8 & 4,4 & 2,6 & 2,8 \\
6,3 & 3,8 & 4,2 & 2,1 & 4,1 & 2,2 \\
\hline 26,5 & 20,0 & 21,4 & 19,2 & 16,5 & 15,1
\end{tabular}

Um 12 Uhr 45 Min. auf 2,89 l, gegenüber 2,81 l der Zeit vor der Aufnahme des Weines. Ich war während der ganzen Zeit ruhig liegen geblieben, anfangs gar nicht, wurde ich erst später schläfrig und schlief beim letzten Nale gleich zu Anfang ein und wurde erst am Schlusse. wach. Trotzdem resultirte die schon oben erwähnte $Z$ ahl als Mittelwerth.

Es schien von Interesse, gleichzeitig auch die Einwirkung des Xeresweines auf den Blutdruck zu untersuchen. Das geht beim Menschen mittelst des Sphygmomanometers von Basch. Gegen dieses Instrument und die bei seiner Anwendung gezogenen Schlüsse lässt sich Manches sagen und ist auch gesagt worden. Allein das dürfte doch zutreffend sein, dass es zu vergleichenden Untersuchungen zur selben Zeit und an derselben Person sich wohl eignet.

Allerdings ist die Einübung mit diesem Instrument nicht leicht, und nach einer bei v. Leube in Würzburg erschienenen DoctorArbeit $^{1}$ ) bedarf man einer vierteljährigen täglichen Uebung, um es zu beherrschen und genaue Untersuchungen mit ihm anzustellen.

1) B. Bozidar, Messung des Blutdrucks am Menschen mit Hülfe des Sphygmomanometers vón Bas ch. Würzburg 1896 . 


\section{Versuch V.}

Der Blutdruck wurde von uns auf der Arteria radialis gemessen und betrug hier $130 \mathrm{~mm}$ Quecksilber, die Athemgrösse 3,21 $\mathrm{l}$.

$10 \mathrm{Uhr} 5 \mathrm{Min}$. trank ich $50_{\mathrm{i}}^{\mathrm{ccm}}$ Xereswein und blieb ruhig liegen:

11 Uhr 30 Min. Blutdruck $160 \mathrm{~mm}$ Athemgrösse 3,30 1 .

$11 " 50 \quad$ \# $170 \quad \# \quad, \quad 3,361$ l.

Mithin war zugleich mit dem Steigen der Athemgrösse auch eine Vermehrung des Blutdruckes eingetreten.

In einem zweiten Versuch über das Verhältniss des Blutdruckes gelangten wir zu folgendem Ergebniss:

\section{Versuch VI.}

Mittlere Athemgrösse vor Einnahme des Weingeistes 3,10 l.

9 Uhr Blutdruck $130 \mathrm{~mm}$. Nach Einnahme:

9 Uhr $15 \mathrm{Min}$. Athemgrösse $3,46 \mathrm{l}$ Blutdruck $145 \mathrm{~mm}$.

\begin{tabular}{|c|c|c|c|c|c|c|}
\hline 9 & 40 & n & $n$ & 3,861 & $\eta$ & $160 \mathrm{~mm}$. \\
\hline 10 & $\eta 20$ & ” & $n$ & 3,361 & $n$ & $150 \mathrm{~mm}$. \\
\hline 10 & 50 & 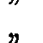 & $\eta$ & 3,351 & $\eta$ & $150 \mathrm{~mm}$. \\
\hline
\end{tabular}

Die Frequenz des Pulses änderte sich nicht regelmässig. Einmal blieb sie sich ziemlich gleich, einmal stieg sie von 72 auf 84 , und das dritte Mal sank sie auf 64 .

Um zu erfahren, wie der Wein bei nü chternem Magen wirke, frühstückte ich an einem Morgen einmal gar nichts, nachdem ich am Abend des vorhergehenden Tages Thee getrunken und einige Butterbrote gegessen hatte.

\section{Versuch VII.}

Ich begann morgens $7 \mathrm{Uhr} 30 \mathrm{Min}$. den Versuch. Mittelwerth der physiologischen Athemgrösse betrug 3,10 l für die halbe Minute. Der Blutdruck, mit dem Sphygmanometer gemessen, zeigte $140 \mathrm{~mm}$. Die Zimmertemperatur betrug hier $22^{\circ} \mathrm{C}$. Un $8 \mathrm{Uhr} 25$ Min. nahm ich $75 \mathrm{ccm}$ Xereswein, wartete wiederum und notirte nach einer Viertelstunde die Werthe:

8 Uhr $40 \mathrm{Min}$. Blutdruck $190 \mathrm{~mm}$ Athemgrösse 4,20 l.

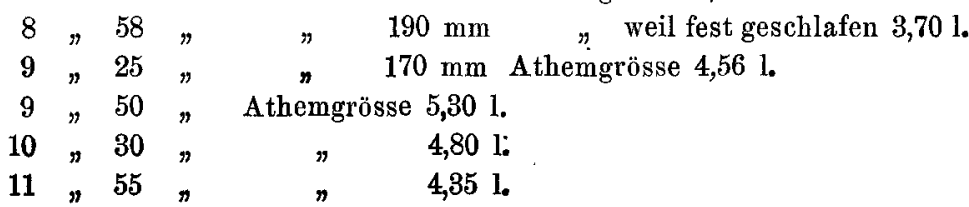

Es ergeben hier die Lesungen das merkwürdige Resultat, dass bei Inanition die Wirkung des Südweines eine ungleich energischere E. Pflúger, Archiv für Physiologie. Bd. 71. 
war als im Zustande der Sättigung. Fin Blick auf die Zahlen genügt, um dies zu zeigen.

Vor der Aufnahme des Weingeistes hatte ich ein unangenehmes Hungergefühl und ein Gefühl allgemeiner Schwäche, was jedoch durch den Weingenuss vorübergehend verschwand.

Ich kann mir das nur so erklären, dass die verbremnbaren Bestandtheile des Weines die nicht vorhandenen gewohnten Nährsubstanzen eine Zeit lang ersetzten.

Auch hier haben wir wieder die Thatsache vor uns, dass innerhalb der 4 stündigen Beobachtungszeit ein Rückschlag gegenüber der Steigerung der Athemgrösse nicht erfolgte.

Ich füge hier die Curve des Versuchs VII (S. 67) an, weil sie in besonders klarer Weise die Wirkung des Weines zeigt.

\section{Versuch VIII.}

Eine andere Versuchsperson, J. G. cand. med, 21 Jahre alt, $65 \mathrm{~kg}$, untersetzt, gesund und kräftig, sehr mässiger Trinker.

Seine normale Athemgrösse betrug im Mittel 2,15 und 2,20 $\mathrm{l}$.

Um 8 Uhr 45 Min. Aufnahme von $60 \mathrm{ccm}$ Xereswein.

\begin{tabular}{|c|c|c|}
\hline $9 \mathrm{Uhr}$ & & Athemgrösse \\
\hline $10 "$ & & $"$ \\
\hline 10 & 45 Min. & $\eta$ \\
\hline
\end{tabular}

12 Uhr 10 Min., 31/2 Stunde nach der Aufnahme des Weines 2,49 l.

Ueberblicken wir die Ergebnisse der bisherigen Versuche, so zeigt sich dies:

1. Nach mässigen Gaben des genossenen Weines stieg die Athemgrösse ganz deutlich.

8. Diese Steigerung war auch dann noch sichtbar, wenn unter dem Einfluss des Weines Schläfrigkeit oder Schlaf eintrat.

3. Innerhalb der benutzten Zeit bis zu vier Stunden war eine die Athemgrösse erniedrigende Rückwirkung nicht wahrzunehmen und wurde auch später in meinem Befinden durch nichts angedeutet.

4. Die Steigerung der Athemgrösse war am beträchtlichsten, als der Körper der Versuchsperson durch Mangel an der gewohnten Nahrungsaufnahme geschwächt war.

5. Die nämlichen Gaben des Weines bewirkten eine Steigerung der Herzthätigkeit. 
Versuch VII.

Damit die Curve nicht zu hreit werde, sind die Zeiten, worin, während ich ruhig lag, nicbt gemessen wurde, weggelassen.

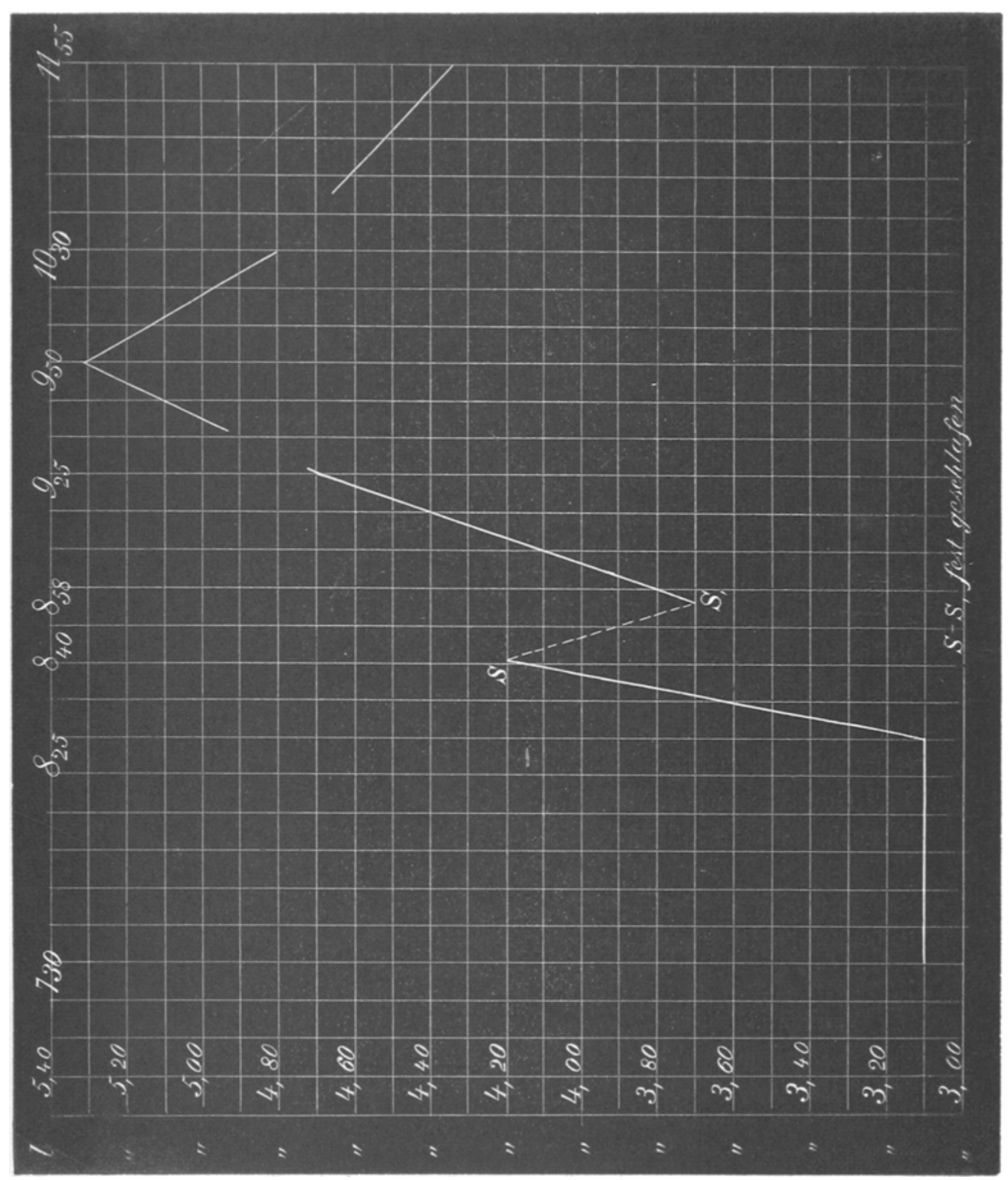


Bei den vorstehenden Versuchen hatte ich nur Gebrauch gemacht von einem bereits 30 Jahre abgelagerten echten Xereswein. Es schien zweckmässig, ein ganz neues Weinpräparat daneben zu prüfen, ich meine den seit wenigen Jahren im Handel befindlichen Malton-Wein.

Er wird bereitet aus gewöhnlicher, sorgfältig hergestellter, aus Gerste gewonnener Bierwüre. Statt dass man dieser Bierhefe hinzufügt, um sie vergähren zu lassen, behandelt man sie zuerst mit Milchsäurehefe in solcher Ausdehnung, dass eine leichte Säuerung daraus hervorgeht. Dann fügt man der so angesäuerten Bierwürze Hefe binzu, die man aus fremden Weinen gewonnen und in der bekannten Bereitung der Kunsthefe weiter gezüchtet hat.

Unter dem Einfluss dieser fremden Hefe wird der grösste Theil des Zuckers - Rohrzucker war noch eigens binzugesetzt worden zu Aethylalkohol und Kohlensäure vergohren. Gleichzeitig aber entwickeln sich diejenigen Aetherarten, die dem Malton-Wein den charakteristischen Geschmack und Geruch der Weine verleihen, wovon die Hefe entnommen und weiter gezüchtet war.

Es zeigt sich also hier die merkwürdige Thatsache, dass die sogenannten Bouquets der natürlichen Weine abhängig sind von der Art der an den Trauben schmarotzenden Hefe.

Das wird heute verwerthet, um sogenannte Malton-Weine im Grossen zu bereiten.

Mir stand ein so bereiteter Xeres-, Tokayer- und Portwein zur Verfügung. Ich wählte den letzteren, als den am besten schmeckenden. Sein Geschmack und Geruch war in der That ein sebr angenehmer, nur bei besonderer Aufmerksamkeit an den Nachgeschmack des Gerstenmalzes erinnernd. Dieser künstliche Portwein enthielt gegen 14,9 Gewichtsprocent Alkohol. Sein süsser Geschmack bewies, dass er noch ein beträchtliches Quantum unvergohrenen Zuckers hatte.

\section{Versuch IX.}

10 Uhr 15 Min. Morgens. Normale Athmung im Mittel 3,15 1 für die halbe Minute. $10 \mathrm{Uhr} 40 \mathrm{Min}$. Aufnahme ron $50 \mathrm{ccm}$ Maltonwein.

10 Uhr 52 Min. Athemgrösse 3,50 1.

Versuch abgebrochen.

$$
11 " 20 \text { " } \quad 3,661 \text {. }
$$

\section{Versuch $\mathbf{X}$.}

9 Uhr 19 Min. Morgens. Normale Athmung 3,20 1. 9 Uhr 42 Min. Aufnahme von $75 \mathrm{ccm}$ Maltonwein.

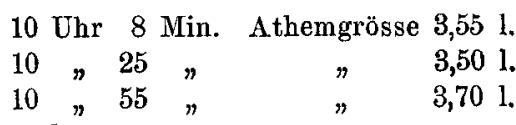

Versuch abgebrochen. 
In diesen beiden Versuchen ergab sich nach Aufnahme des Malton-Weines eine deutliche Reizung der Athmungscentren, allerdings nicht in dem Umfange, wie bei dem alten Xereswein. Ich prüfte desshalb im folgenden Versuch dieselbe Wirkung nach Aufnahme grösserer Gaben und fand dabei dieses:

\section{Versuch XI.}

9 Uhr 40 Min. Morgens. Normale Athmung:

\begin{tabular}{rrrrrr}
3,3 & 3,5 & 3,1 & 3,0 & 3,4 & 2,9 \\
3,5 & 3,3 & 3,2 & 3,0 & 2,8 & 2,5 \\
3,0 & 2,3 & 3,5 & 3,5 & 3,0 & 3,0 \\
3,4 & 2,6 & 2,9 & 3,2 & 2,6 & 3,1 \\
3,4 & 2,8 & 3,6 & 3,4 & 3,4 & 3,0 \\
\hline 16,6 & 14,5 & 16,3 & 16,1 & 15,2 & 14,5
\end{tabular}

Im Mittel 3,10 I für die halbe Minute.

10 Uhr. $75 \mathrm{ccm}$ Maltonwein.

10 Uhr 10 Min. folgende Athemgrösse:

\begin{tabular}{rrrrrr}
3,4 & 4,0 & 3,5 & 4,3 & 3,4 & 3,8 \\
3,8 & 4,0 & 3,5 & 4,2 & 3,9 & 3,7 \\
3,8 & 3,7 & 3,8 & 4,5 & 3,7 & 3,5 \\
4,0 & 3,3 & 4,2 & 4,3 & 4,0 & 3,6 \\
3,7 & 4,0 & 4,0 & 4,0 & 4,2 & 3,3 \\
\hline 18,7 & 19,0 & 19,0 & 21,3 & 19,2 & 17,9
\end{tabular}

Im Mittel 3,80 I für die halbe Minute.

10 Ohr 30 Min.:

\begin{tabular}{rrrrrr}
3,5 & 3,7 & 3,4 & 3,6 & 2,5 & 3,0 \\
4,0 & 4,0 & 3,3 & 3,2 & 2,7 & 3,0 \\
4,2 & 3,7 & 4,0 & 3,5 & 2,9 & 3,2 \\
4,6 & 3,0 & 3,2 & 3,3 & 3,1 & 3,4 \\
3,8 & 3,3 & 3,2 & 3,0 & 4,1 & 2,9 \\
\hline 20,1 & 17,7 & 17,1 & 16,6 & 15,3 & 15,5
\end{tabular}

In Mittel 3,30 1 für die halbe Minute.

$10 \mathrm{Uhr} 50 \mathrm{Min} .75 \mathrm{ccm}$ Wein.

11 Uhr:

\begin{tabular}{rrrrrr}
3,0 & 4,0 & 3,8 & 3,1 & 3,2 & 3,7 \\
3,3 & 3,4 & 3,7 & 3,2 & 3,5 & 3,3 \\
3,0 & 3,3 & 3,2 & 3,5 & 3,0 & 3,4 \\
3,5 & 3,5 & 3,8 & 3,4 & 3,5 & 3,4 \\
3,3 & 3,5 & 3,7 & 3,2 & 4,0 & 3,4 \\
\hline 16,1 & 17,7 & 18,2 & 16,4 & 17,2 & 17,2
\end{tabular}

Im Mittel 3,40 l für die halbe Minute. 
$11 \mathrm{Uhr} 30 \mathrm{Min}$. Nochmals $75 \mathrm{ccm}$ Maltonwein.

11 Uhr 40 Min.:

\begin{tabular}{rrrrrr}
4,5 & 4,8 & 4,5 & 4,4 & 4,0 & 4,0 \\
3,5 & 4,2 & 4,2 & 3,9 & 3,8 & 4,0 \\
4,0 & 4,0 & 4,3 & 4,0 & 4,0 & 4,2 \\
3,5 & 4,3 & 4,0 & 3,8 & 4,0 & 4,6 \\
4,0 & 4,7 & 4,1 & 3,7 & 3,9 & 4,2 \\
\hline 19,5 & 22,0 & 21,1 & 19,8 & 19,7 & 21,0
\end{tabular}

Im Mittel 4,10 1 für die halbe Minute.

12 Uhr Mittags:

\begin{tabular}{rrrrrr}
$\mathbf{3}, 7$ & 4,3 & 4,5 & 4,0 & 4,0 & 4,2 \\
4,2 & 4,5 & 4,5 & 4,0 & 3,9 & 4,1 \\
3,8 & 4,1 & 4,5 & 4,2 & 4,3 & 3,9 \\
4,1 & 4,0 & 3,9 & 4,1 & 4,5 & 4,2 \\
$\mathbf{4 , 6}$ & 4,0 & 4,1 & 4,0 & 4,2 & 4,5 \\
\hline 20,4 & 20,9 & 21,5 & 20,3 & 20,9 & 20,9
\end{tabular}

Im Mittel 3,83 1 für die halbe Minute.

Versuch abgebrochen.

Wie man sieht, ist eine recht gute Reizwirkung vorhanden. Sie erfordert allerdings eine grössere Aufnahme als bei dem alten Xereswein.

Mein Befinden nach Aufnahme des echten Xeresweines war folgendes: Zuerst angenehme Erregung des Allgemeingefühls, dann Schläfrigkeit, die einige Male in vollen tiefen Schlaf überging. Keinerlei unangenehme Nachwirkung.

Beim Malton-Wein: Die ebenerwähnte Steigerung des Allgemeingefühls war nicht vorhanden, aber auch nicht die Schläfrigkeit und der Schlaf, ungeachtet die Gabe grösser war als beim Xeres; jede üble Nachwirkung fehlte.

Auch der Malton-Portwein also erwies sich bei mir als ein Getränk, welches die Athmungscentra ohne störende Neben- oder Nachwirkung erregte. Es muss Gegenstand weiterer Untersuchungen sein, ob das, auch bei andern Personen die Regel ist.

Im Uebrigen verweise ich betreffs seiner Anwendung am kranken Menschen auf die Versuche von C. A. W. Fwald, Berlin. Klin. Wochenschr. $1895 \mathrm{Nr}$. 45. - M. Mendels ohn, Zeitschr. für Krankenpflege. Juniheft 1895. - C. v. Noorden, Berlin. Klin. Wochenschr. 1897 Nr. 44.

Aus der letztgenannten Veröffentlichung will ich nur folgenden Satz hervorheben: 
„Der günstige Einfluss der Maltonweine auf das Allgemeinbefinden, die anregende Wirkung auf das erschöpfte Nervensystem, die Anspornung der Herzthätigkeit vollzog sich in derselben Weise, wie bei anderen Weinen von ähnlichem Alkoholgehalt."

Legen wir uns die Frage vor, welche Bestandtheile der von mir geprüften Weine die Träger der Wirkung waren, so müssen als solche genannt werden, gemäss den früheren Versuchen, der Aethylalkohol und die Säureäther; ob nicht noch andere, wie die Extractivstoffe u. s. w. muss die weitere Untersuchung ergeben.

Sollten die Ergebnisse meiner Versuche Verwendung am Menschen finden, so kann das nur an dem durch Krankheit oder Arbeit erschöpften sein, denn der gesunde Mensch hat kein Reizmittel für seine Athmungs- und Herzthätigkeit nöthig.

Die Arbeit wurde auf Anregung und unter Anleitung von Geh. Med.-Rath Prof. Dr. Binz unternommen. Hierfür spreche ich meinem hochverehrten Lehrer meinen Dank aus. Desgleichen danke ich dem Privatdocenten Dr. Wendelstad t für seine überaus grosse Freundichkeit, mit der er mich bei den zahlreichen Versuchen unterstützt hat. 